\title{
L'Utilisation des eaux en excès pour renforcer les chutes d'eau.
}

Nous recevons d'un de nos lecteurs une lettre dont nous extrayons le passage suivant:

"Dans la note de M. A. Foch sur l'utilisation des eaux en excès pour renforcer les chutes, il est dit, " page $58,2^{\mathrm{e}}$ colonne, qu'il serait à désirer que les résultats signalés soient bientôt contrôlés en France.

"Il y a plus de quinze ans que des expériences en grand ont été faites sur un dispositif un peu différent, " mais conduisant au même but. Je vous adresse une brochure qui rend compte de ces expériences".

Nous publions ci-après un résumé des résultats obtenus. Il sera intéressant, croyons-nous, de pouvoir les comparer à ceux que pourra publier ultérieurement la" "General Electric Review" .

Pour remédier à cette situation paradoxale d'une usine hydraulique qui rend d'autant moins que le débit de la rivière est plus puissant, M. M. Saugey, chef de service à l'Usine de Chèvres, imagina un système d'installation basé sur le fait hydraulique suivant que le niveau d'une nappe d'eau comprise entre 2 jets horisontaux, coulant en vitesse, est abaissé par l'action aspirante de ces jets. En particulier, si dans un barrage on ouvre deux vannes non contiguës, les deux jets qui en sortent abaissent le niveau dans l'espace intermédiaire ; l'aspiration des jets latéraux creusant entre ceux-ci, en aval du barrage une poche qui ne se comble pas, même quand on y verse constamment de l'eau. De cette remarque découle tout naturellement le dispositif employé qui consiste à faire déboucher la conduite d'aspiration de la turbine entre les deux jets puissants envisagés.

Des expériences et des mesures ont été faites sur les deux principaux cours d'eau du canton de Genève : le Rhône, à Chèvres, et l'Arve à Vessy.

\section{Expériences de Chèvres.}

Elles remontent à la période d'été de l'année 1905. Le Rhône roulait alors 800 mètres cubes d'eau à la seconde, ce qui a permis de faire des essais absorbant plusieurs centaines de mètres cubes sans nuire à la bonne marche de l'usine.

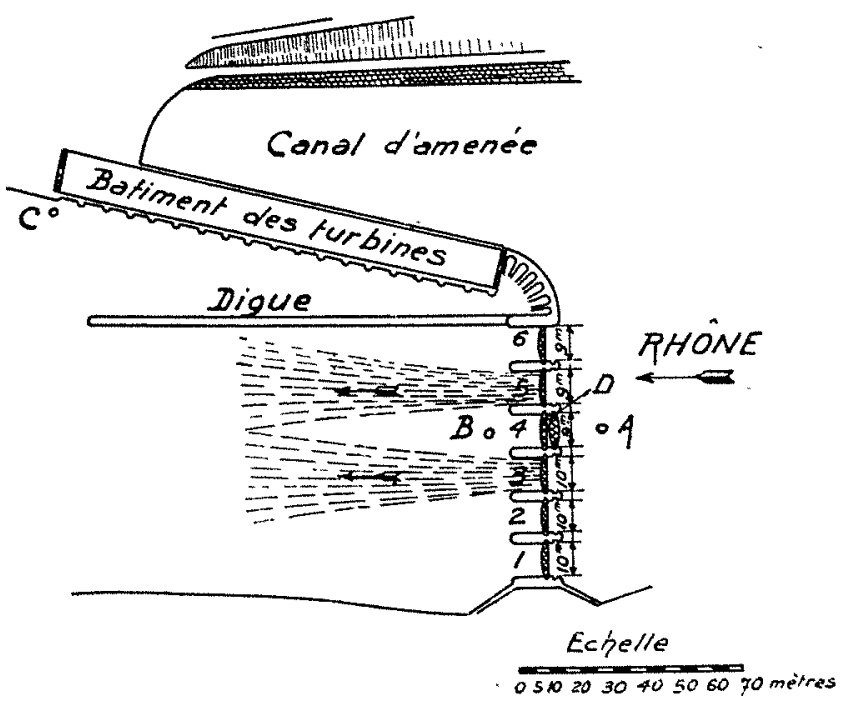

Fìg. 1. - Disposition générale des expériences faites à l'usine de Chèvres, près Genève.

\section{TEGENDES}

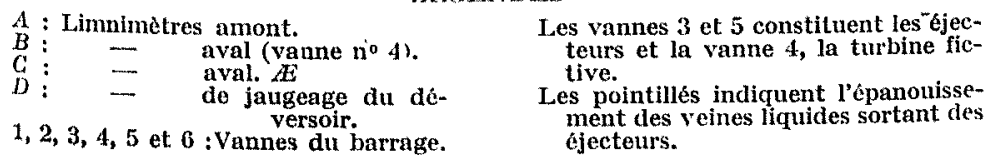

La lisposition de l'usine de Chèvres est représentée sur la fi- gure 1. Pour l'expérience les jets aspirants étaient fournis par les vannes 3 et 5 . Le niveau artificiel se trouvait ainsi produit '̀̀ l'aval de la vanne 4 . Trois limimimètres $\mathrm{A}, \mathrm{B}, \mathrm{C}$, indiquaient les trois niveaux intéressants à connaître ; à savoir : le niveau amont (en A), le niveau aval naturel (en $\mathrm{C}$ ) ; le niveau aval artificiel (en B). E'expérience consista à mesurer simultanément les trois niveaux $\mathrm{A}, \mathrm{B}, \mathrm{C}$, pendant que les vannes 3,4 et 5 débitaient des quantités d'eau très variables. La vanne 4 représente ici une turbine fictive.

Nous ne voulons pas entrer dans le détail des expériences. Signalons simplement que l'opération la plus délicate a été la mesure du débit de la vanne 4 : il était nécessaire que ce débit fût maintenu constant, et que l'eau sortît de cette vanne à une vitesse aussi réduite que possible, ce qui correspond en effet aux conditions d'une bonne installation hydraulique.

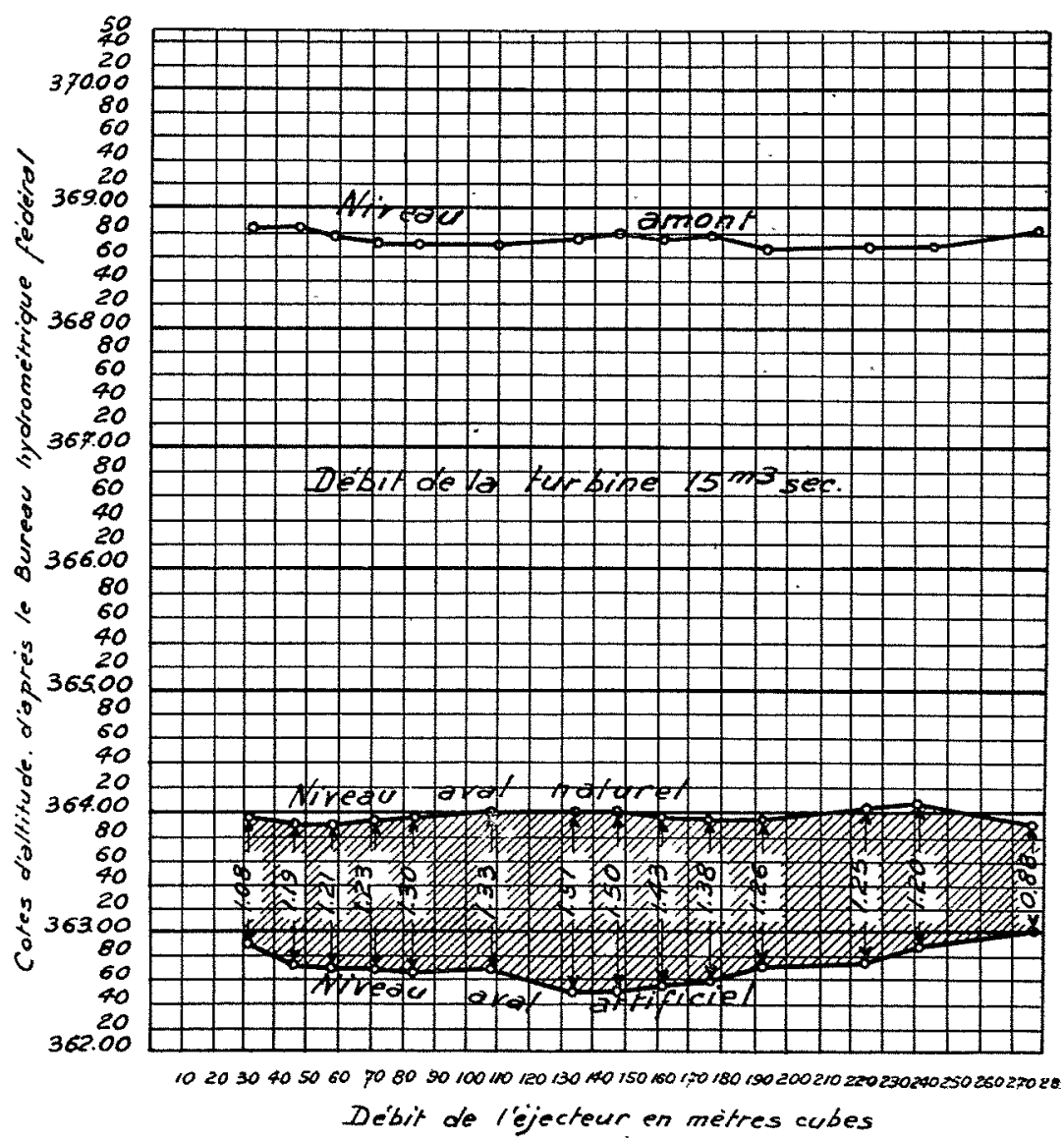

Fig. 2. - Essais au Barrage de l'Usine de Clières, le 18 juin 1905.

$1^{\text {re }}$ Expérience. - Le débit de la turbine fictive étant maintenu constant on a fait varier celui des éjecteurs 3 et 4 . Les résultats sont consignés dans le diagramme faisant l'objet de la ifgures 2. 


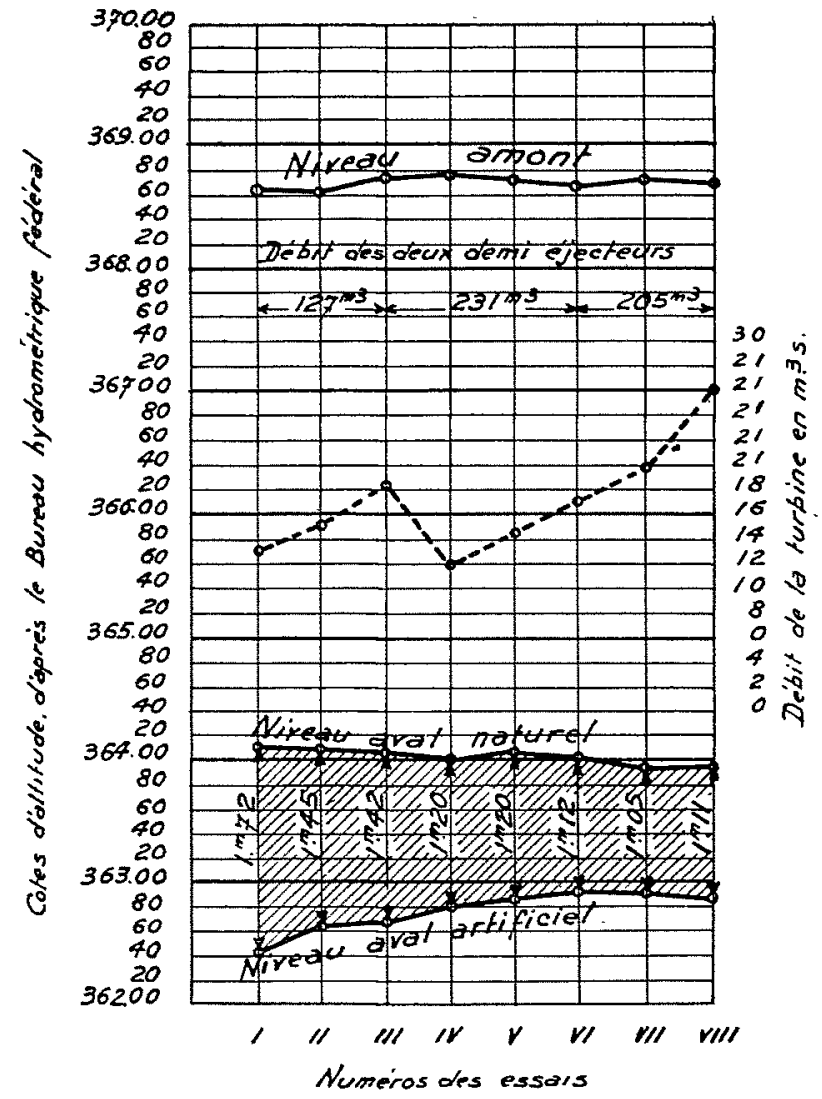

Fig. 3. - Essais au Barrage de l'Usine de Chèvres, le 16 juillet 1905.

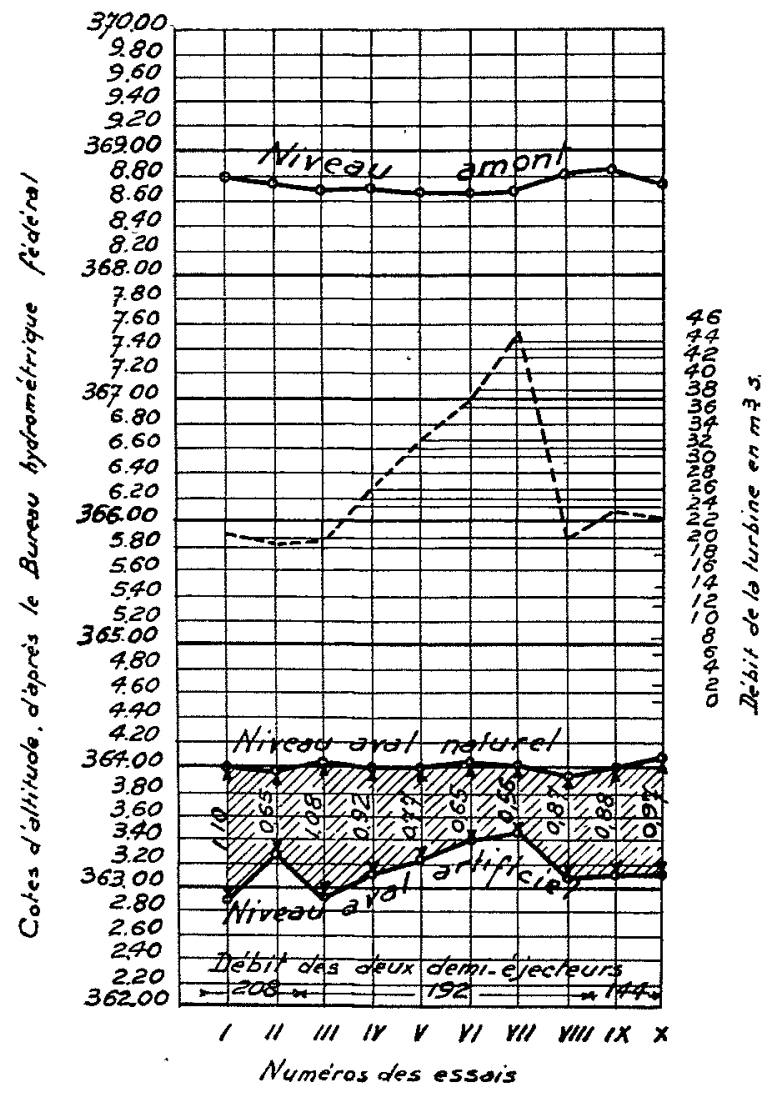

2e Expérience. - Le débit des vannes 3 et 5 étant maintenu constant, on a fait varier celui de la turbine fictive (vanne 4). Cet essai a été répété pour 3 valeurs du débit des vannes 3 et 5, savoir : 127, 231 et 205 mètres cubes par seconde. Les résultats de ces essais sont résumés par le diagramme 3 .

3e Expérience. - On a répété des expériences analogues à celles dont il vient d'ètre fait mention, mais en utilisant comme éjecteurs les vannes 2 et 6 . L'espace intermédiaire, siège de la dénivellation artificielle, était alors d'une largeur de $42 \mathrm{~m}$. 50. Les vannes 3 et 4 jouaient le rôle de turbines.

Les résultats de ces essais font l'objet du diagramme 4.

Essais à l'Usine de Vessy-sur-Arve, le 26 août 1905.

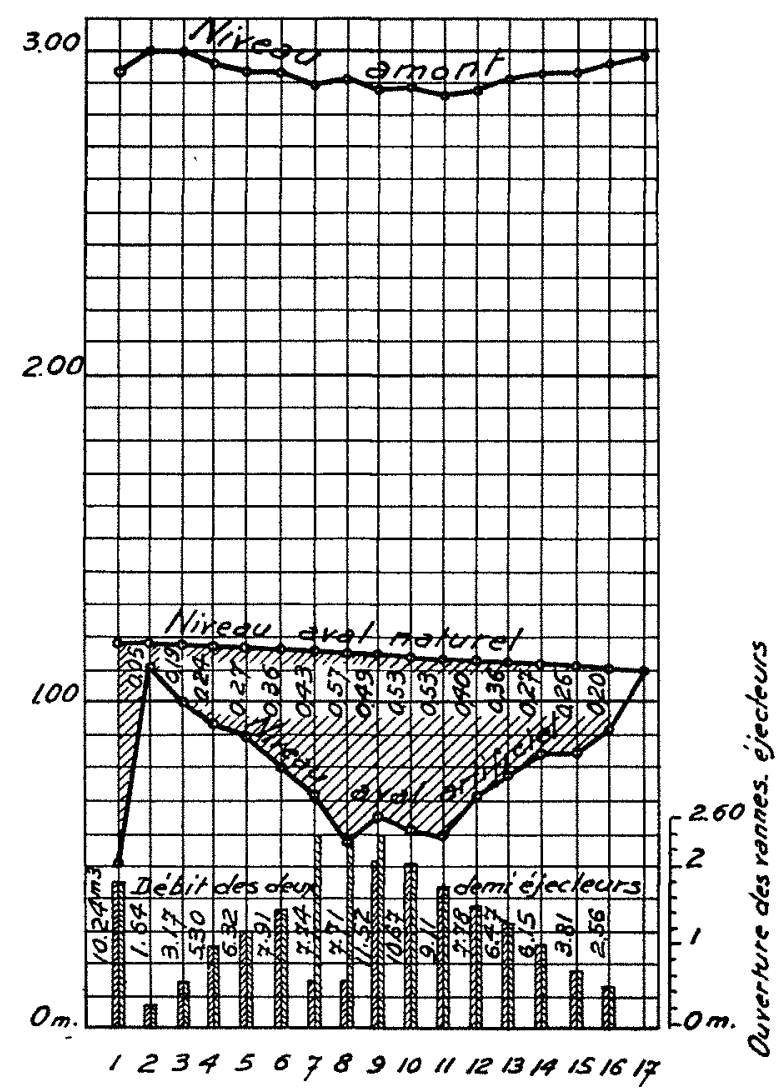

Fig. 5. - Graphique donnant la chute récupérée, en fonction du débit des éjecteurs. Débit de la turbine : 1660 litres par seconde.

\section{EXPERIENCES DE VESSY.}

Les expériences effectuées à l'usine de Vessy sur l'Arve ont été beaucoup plus réduites que celles de Chèvres.

Une chambre de turbines a été installée entre 2 vannes extrêmes d'un barrage de décharge. Plusieurs séries d'expériences ont été entreprises à l'époque des crues d'été, la chute du barrage n'atteignant guère qu'un mètre et demi, alors qu'en hiver elle était du double. Le débit de la túrbine était de 1660 litres à la seconde, et celui des éjecteurs s'élevait à environ 10.000 litres.

On a fait une série de mesures en variant le débit des éjecteurs. Afin de juger de l'effet de la dyssymétrie, on a également effectué quelques mesurès pour les débits inégaux des deux éjecteurs. Les résultats de ces essais sont consignés sur le graphique faisant l'objet de la figure 5 .

Fig. 4. - Essais au Barrage de l'Usine de Chèvres, le 16 juillet 1905. 